\title{
Iterates of a Number-Theoretic Function
}

\author{
By Mohan Lal*
}

Abstract. Iterates of a function defined by the sum of the prime divisors of a number, where the multiple factors are counted multiply, are considered. The process of iteration is terminated at a prime. The density distribution of these primes is investigated empirically, for $N \leqq 60000$ and it is found to be quite constant.

Introduction. Let $n=\prod_{i} p_{i}{ }^{\alpha_{i}}$ be the representation of $n$ as a product of distinct primes and define a function,

$$
J(n)=\sum_{i} \alpha_{i} p_{i}
$$

Thus the function $J(n)$ is defined to be the sum of the prime divisors of $n$ and the multiple factors are counted multiply. From (1) it follows that $J(n)$ is completely additive, for $J(m n)=J(m)+J(n)$.

For $n$, a prime, $J(p)=p$ and for $n$, a composite number, $J(n)<n$. For $n=4$, $J(4)=4$ and this is considered to be an exceptional case and thus the number 4 behaves like a prime.

The $r$ th iterate of $J(n)$ is defined by

$$
J_{r}(n)=J\left(J_{r-1}(n)\right) ; \quad J_{1}(n)=J(n) .
$$

When $n$ is a prime, each successive iterate gives rise to the same prime and we may say that the process of iteration converges. In what follows we will assign a value of 1 to $r$, for $n=$ prime. For composite numbers, $r$ takes definite positive integral values for the iteration (2) to converge. Thus for $n=8, r=3$ since $J_{1}(8)=6$; $J_{2}(8)=5 ; J_{3}(8)=5$. In this manner, we shall associate with an integer $n$, a function $R(n)$ which defines the minimum number of iterates of $J(n)$ required to transform it into a prime. Thus, we define $R(n)=r$ and so, $R(30)=3, R(24)=4, R(10)=2$.

Naturally the question arises, "What can be said about $R(n)$ ?"

Still, another interesting problem related to the iterates of $J(n)$ could be stated as follows. Suppose we apply the iterates of $J(n)$ to all integers less than or equal to $N$, we obtain a set of primes which are distributed all over the interval. Then, if $n\left(p_{i}\right)$ is the number of primes $p_{i}$ between 1 and $N$ inclusive, "Does the ratio $n\left(p_{i}\right) / N$ approach a definite limit, as $N$ approaches infinity, for all $p_{i}$ ?" It should be stated that the prime $p=3$ occurs only once and $n=4$ is an exceptional case for which $R(4)$ is not defined in our context.

To find analytic solutions to these questions, which involve various partitions of a number into primes, would probably be difficult. In what follows we provide an empirical investigation of these problems.

Received January 12, 1968, revised July 1, 1968.

* This work was partially supported by the National Research Council under the Grant No. A4026. 


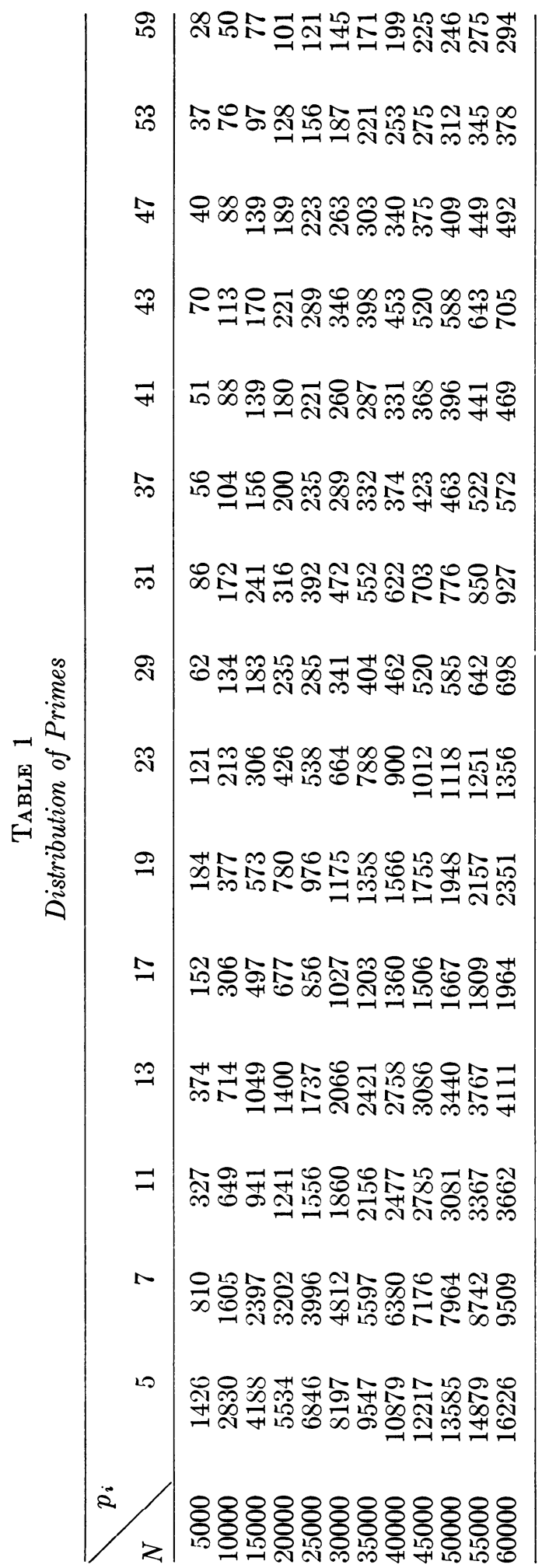

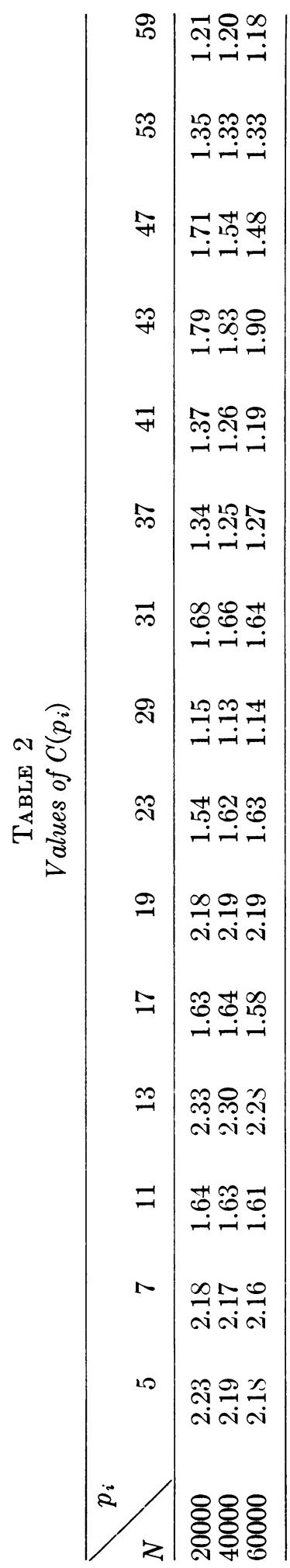


We studied $R(n)$ and $J_{r}(n)$ for $n \leqq 60000$, using a sieve method. In Table 1, we present the number of primes $n\left(p_{i}\right)$, for $5 \leqq p_{i} \leqq 59$ at steps of 5000 . We note that for these 15 values of $p_{i}, n\left(p_{i}\right) / N$ is quite constant. Therefore, there is strong indication that the ratio $n\left(p_{i}\right) / N$ approaches a definite limit, as $N \rightarrow \infty$.

Further empirical study reveals that $n\left(p_{i}\right)$ is very roughly proportional to $N /\left(p_{i} \times \log p_{i}\right)$. This is shown in Table 2 , where we tabulate

$$
C\left(p_{i}\right)=p_{i} \times \log \left(p_{i}\right) \times n\left(p_{i}\right) / N
$$

for $N=20000,40000$ and 60000 . The values of $C\left(p_{i}\right)$ are quite consistent for these three values of $N$.

As far as $R(n)$ is concerned, we note the empirical result that $R(n) \leqq[\log (n)]+3$, for $n \leqq 60000$.

In conclusion, we might indicate, that though (3) describes the distribution $n\left(p_{i}\right)$ approximately, it is not entirely satisfactory, since it does not indicate why $n(p+2)>n(p)$, in the case of twin primes, except for $p=5$. One wonders if the expression (3) has any basis, heuristic or otherwise. The constants $C\left(p_{i}\right)$ do vary considerably for these fifteen values.

Memorial University of Newfoundland

St. John's, Newfoundland, Canada 